\title{
Research on Analysis and Visualization of The DataTransformation Interface Based on Logging While Drilling(LWD)
}

\author{
WANG Peng, ZHANG Yan-mei, Chen Hong-wei \\ School of Information Engineering, China University of Geosciences (Beijing), Beijing 100083
}

Keywords: DataTransformation, Logging While Drilling, Visualization.

\begin{abstract}
In order to solve the problem that the different format data collected from sites are transferred among computer systems, the project finished the modularization for reading the data file brought from field acquisition and the drawing of image and curve, which will be exported to the specified file in the manner of WITS format according to the site data transmission specification. And this could provide more convenience to analysis and judge data information for users. Through the testing of experimental data, the interface is able to meet the design requirements, improve interactivity and practicality. The paper is based on Visual Studio 2008 platform and the program used $\mathrm{CA}$ language with good encapsulation.
\end{abstract}

\section{Introduction}

With the development of electronic technology and computer technology, logging technique has developed into a high and new technology integration of multi discipline and technology integration, which plays an indispensable role in the field of petroleum exploration and development. However, field exploration workers use different types and models of logging instrument and data format has heterogeneity when they are collect data, which bring obstacles between different computer systems and service corporation in the transmission process ${ }^{[1]}$. As a result, with the further development of logging technology, the software that processes data from well logging must be developed. At this time, it is practical significant to uniform data format and methods collecting data to improve the fluency and standardization of data communication.

WITS (Wellsite Information Transfer Specification) as a recommended communication protocol can transmit data in different formats between computer systems in petroleum exploration and development, which is convenient to share and use information and can avoid repetitive work for accomplishing the purpose of improving the work efficiency.

\section{Systems analysis of data transformation}

In the drilling site, the drilling fluid column sends the signal from the sensor to the ground. Then technical workers decode and process the signal by the pressure sensor and the data receiving system, which is stored to computer system according to the specific data format. At last, the source data is stored in the database ${ }^{[2]}$. Specific data acquisition process is shown in Figure1. 




Figure 1. Data acquisition schematic diagram

The source data shows two kinds of information opened with UItraEdit software. The first kind of information is the document information of the source file. File information is a string that is stored in the line unit and each line of information start with '\#'. There is keywords' name and value followed by '\#' and part of keywords has other instructions. File information mainly includes the version information of the document, data type and engineering name. The second kind of information is the data information of the source file, which is stored in the form of binary flow.

WITS is a communication format with clear content and format. Its data stream is composed of a series data sets and every data set starts with "\&\&" and ends with "!!". Every data item consists of one 'Identifier' that represents the type of record data and one 'Value' can be a text string or can be represented by a number of ASCII code ${ }^{[3]}$.

\section{Research on data transformation interface}

In the course of understanding source data format and target data format, accord with actual demand we plan to read the source file information and complete the conversion of the target data format to implement the interface ${ }^{[4-5]}$.

\subsection{Research on reading the source files data}

Before the target data format conversion, we need to extract the information of source files which process need four models to achieve.

(1) Configuration information module. Configuration information is written to the application directly in the XML data format ${ }^{[6]}$. Configuration information contains core content of file information and also provides the necessary condition to parse data as a premise before displaying file information and extracting data information. Part of the configuration information content is shown as Table 1 .

Table1. Header keyword

\begin{tabular}{|c|c|}
\hline Keyword & Description \\
\hline Field & Field \\
\hline DataType & Data type \\
\hline Project & Project name \\
\hline dataSrc & Source data \\
\hline FileID & File label \\
\hline
\end{tabular}

Table 2 . File information

\begin{tabular}{|c|c|c|c|}
\hline Keyword & Description & Value & Data type \\
\hline DataType & Data type & GRCPR & \\
\hline Field & Field & Key_time & Int64 \\
\hline Field & Field & Gr & Double \\
\hline Field & Field & Flags & Int32 \\
\hline Project & Project name & Hang-28H & \\
\hline
\end{tabular}

(2) File information extraction module. File information is used to store field information from binary flow in line with the unit. After the design of the main framework of the basic framework, we can use "keyword", "keyword 
description", "keyword value", "data type" to initialize file header information and display file information. Taking one kind of data as an example, the concrete content is shown in the table 2.

(3) Data information extraction module. Parsing data information is one of the core tasks of the project development, which is based on configuration information and file information. In the process of reading data flow, we create an auxiliary transition table to store temporary data and transition data ${ }^{[7]}$. The flow chart of parsing data information is shown as Figure 2.



Figure 2. Flow chart of parsing data

(4) Visualization of graph and image model ${ }^{[8-9]}$. In order to remove burrs data, parsing data is drawn in graph and image to observe the changes of data for professionals. As achieving visualization function of graph and image, we can mainly use formula 1 and formula. 2 as followed.

$$
\begin{gathered}
\mathrm{d} y=\frac{\text { height }}{\text { count }} *(\text { di }+1) \\
\mathrm{d} x=5+\frac{\text { data }- \text { min_value }}{\left.\max \left(\max _{\text {_value }}-\text { min_value }, 1\right)_{\text {mal }}\right)} * \text { width }
\end{gathered}
$$

In these two formulas, height represents the height of the entire image; count represents the number of the current data rows; data represents the size of the current data; max_value and min_value represent the maximum and minimum values of the selected data respectively; max is a comparison of the size of the function, which is used to compare the difference between the maximum and the minimum value and the value of one. In addition, x-coordinate is added with the value of five so that the whole image distribution is more uniform.

\subsection{Research on data format conversion}

Data format conversion is another key task of the development. Its purpose is that parsing data is converted into the target data in WITS format for production practice in the use of fast and convenient and this process need three models to 
achieve.

(1) Navigation information module. Navigation information contains some identifier items that come from the WITS specifications, which is used as a small database for data format conversion model. We can simulate some identifier items as shown in table 3.

Table 3 Navigation information

\begin{tabular}{|c|c|c|c|}
\hline One level code & Primary heading & Two-level code & Secondary heading \\
\hline 01 & General Time-Based & 03 & Key_Time \\
\hline 01 & General Time-Based & 02 & Flags \\
\hline 02 & Drilling Depth Based & 04 & Depth \\
\hline 03 & Drilling Connection & 11 & Temperature \\
\hline 07 & Survey Directional & 12 & SURV \\
\hline 08 & MWD Form Evaluation & 10 & Gr \\
\hline 08 & MWD Form Evaluation & 07 & CPR \\
\hline 08 & MWD Form Evaluation & 09 & Rop \\
\hline$\ldots$ & $\ldots$ & $\ldots$ & $\ldots$ \\
\hline
\end{tabular}

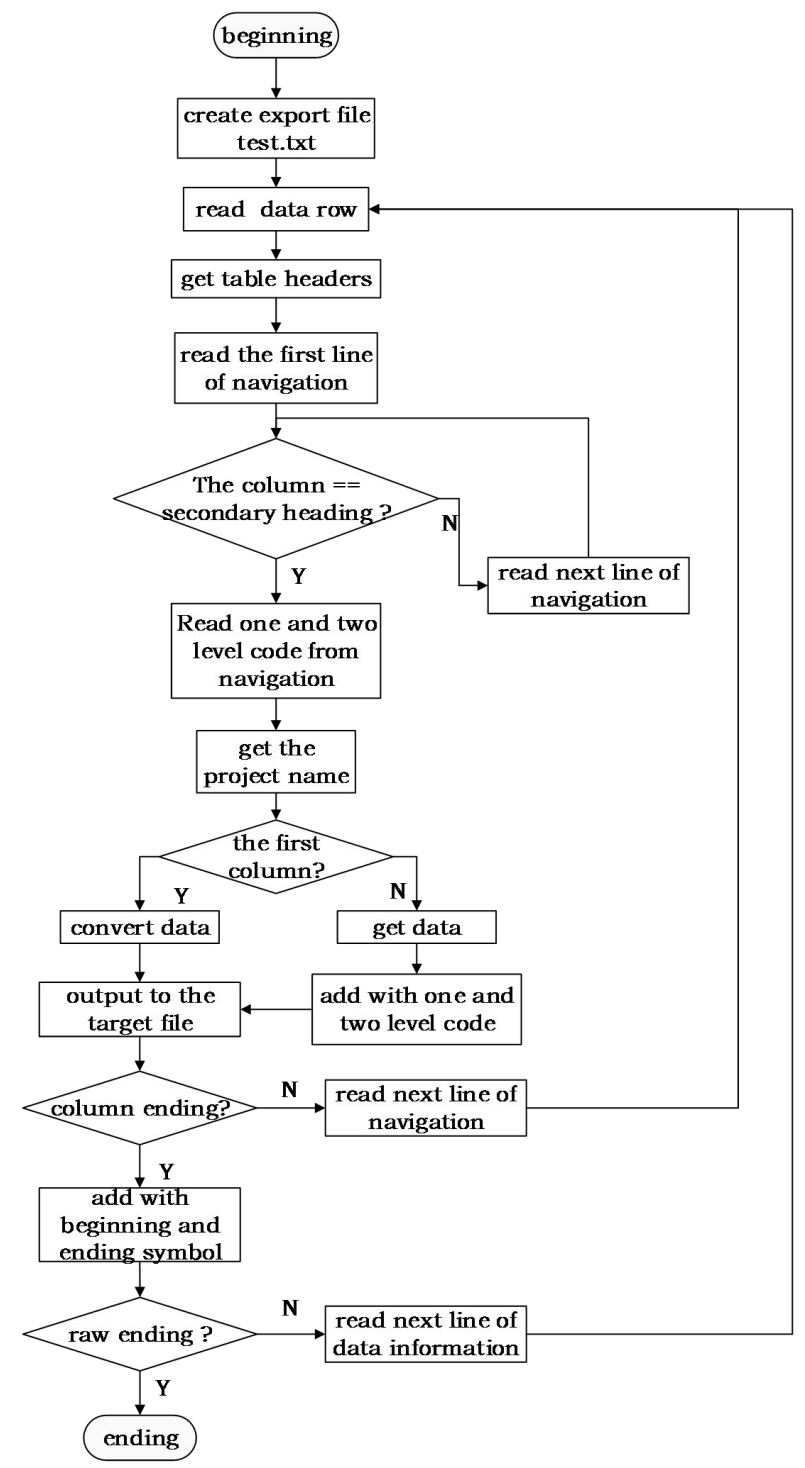

Figure 3. Flow chart of data format conversion

(2) Data matching module. Data matching is that some data element tables with a common data item is combined 
into a new table of data with greater amount of information and more practical. Professionals can choose and deal with required data information according to the actual need. The new data table improve the use efficiency of data greatly.

(3) Data format conversion model o The purpose of data format conversion is to extract the common information from the data table of the navigation information, file information data tables and data tables for combination and processing. At last, the entire data sets in WITS format are formed. In the process of data conversion, we repeatedly used to nest, loop and traverse ${ }^{[10]}$. The flow chart of data format conversion is shown as Figure 3.

\section{Conclusion}

The project implements the interface that converts the data format that is based on logging while drilling. The interface can parse all information of source file and converts it into WITS format that is approved by the oil industry. The interface makes it easy to share and transmit data and improve the work efficiency of practical production. However, while the interface is in operation, the program runs relatively slow. Therefore, the next step is to optimize the code quality and improve the operation speed ${ }^{[11-13]}$.

\section{Acknowledgement}

This paper is supported by National Natural Science Foundation of China (91328201).

\section{Reference}

1. Li Yuhuan. Measurement is The Only Way for The Development of Mud Logging Industry - Technical Ideas About The Drilling Mud Logging [J]. Mud Logging Engineering,2006,17(1):36 38.

2. Wang Ran, Liu Ziying, Zhang Youguang. Design and Implementation of Remote Data Transmission System for Petroleum Logging [J]. Computer Engineering, 2006,32(4):236 238;

3. Cha Xiuqi, Wu Rongquan, Gao Yuanjun. Research on The Technology of C/S to B/S Mode Conversion [J]. Computer Engineering,2014,40(1):263 267.

4. Zhang Xinxin. Research on The Technology of Data Migration and Conversion Interface of Comprehensive Logging Instrument [D]. Dong Ying: China University of Petroleum (Hua Dong), 2008.

5. Dai Yongshou, Zhang Shuying. Development of Data Conversion Interface Technology Based on JAVA and XML [J]. Mud Logging Engineering,2008,19(2):65 67.

6. David Hunter Jeff Rafter Joe Fawcett. XML Entry Classic [M]. Wu Wenguo. 4. Beijing: Tsinghua University Press, 2009.

7. Wang Huiping, Wang Wei. Automatic Identification and Conversion Method of Well Logging Data Format Based on Feature Database [J]. Well Logging Engineering,2014,38(1):65 68.

8. Shu Huizhen, Lin Caixia. Reading and Drawing of Log Data [J]. Oil Instrument,2008,22(4): 82 84.

9. Zhang Wendong, Huang Chao, Pan Yunyan. Design and Implementation of a Pipe String Drawing System Based on Flex [J]. Computer Engineering,2012,38(21):237 240.

10. Gong Fuxiu. Development of Data Format Conversion System [J]. Jianghan Petroleum Science and Technology,2006,16(1):65 68.

11. Stephen R.Schach. Object Oriented and Traditional Method of Software Engineering [M]. Deng Yingchun, Xu Tianshun. 7. Beijing:: Machinery Industry Press, 2007.

12. Ron Patton. Software Testing [M]. Zhang Xiaosong, Wang Yu, Cao Yue.2.Beijing: Machinery Industry Press,2009.

13. Karli Watson Christian Nagel. C\# Entry Classic[M]. Qi Libo. 5. Beijing: Tsinghua University Press, 2010. 